\title{
PAST, PRESENT AND FUTURE
}

In this concluding chapter we first discuss the historical background with respect to the material presented in chapters II through IV. Second, we discuss some of the results which have not been presented in these chapters pointing out relevant papers and reports in the Bibliography. Finally some new directions and open problems are mentioned. 


\section{V.1 Historical Background}

In this first section we discuss the origins of the material presented in chapters II, III and IV. Comments on new and/or related results are also included where appropriate.

Chapter II: Grammar forms and what we have called g-interpretations were introduced by Armin Cremers and Seymour Ginsburg in their conference paper, Gremers and Ginsburg [1974], which subsequently appeared as cremers and Ginsburg [1975]. This approach was preceded by an attempt of Gabrielian and Ginsburg [1974] using grammar schemata and the thesis of Workman [1974] who defined families of grammars by descriptive complexity measures. The notion of an s-interpretation was first introduced in Bertsch [1975], but the definition given there is incorrect. A correct definition was subsequently given in Ginsburg and Mayer [1976], while related work is to be found in Maurer and Wood [1976], Nivat [1976] and Walter [1976]. The discussion of closure properties for collections of grammars running through the examples for Section II.1 follows the opening discussion in Greibach [1977].

The study of collections of grammar families was initiated in Ginsburg and Maurer [1976] and the original notion of quasiinterpretation is to be found in Ginsburg and Maurer [1978]. It should be noted that "quasi-interpretation" in the Ginsburg and Maurer [1978] sense is what in Sections II.3.1 and II.3.2 is called a "full quasiinterpretation". The composition results of Section II.3.2 are taken partly from Ginsburg and Maurer [1978] and mainly from von Braunmuh1, Hotzel and Wood [1980]. The results of Sections II.3.3 and II.3.4 were originally proved by Ginsburg and Maurer [1976].

The reduction results and normal form theorems for sinterpretations in Section II.4.1 are either new or taken from Maurer, saloma and Wood [1980b]. The "super-normal form" theorem was discovered independently by Blattner and Ginsburg [1977] and Maurer, Salomaa and Wood [1980a]. The extension of this theorem to $\mathrm{g}$ grammatical families was however the work of Blattner and Ginsburg [1977 and 1980]. The presentation followed in Section II.4.1 is however new. The results for g-interpretations are taken from cremers and Ginsburg [1975] as are the closure results and the principality result (Section II.4). Further work on normal forms can be found in Cremers, Ginsburg and Spanier [1977 and 1979]. That every finite g-grammatical family is s-grammatical was first proved in vashnavi and Wood [1978]. The characterization of when $\mathcal{L}_{S}(G, \Rightarrow)=\mathscr{L}(R E G)$ or $\mathcal{Z}$ (LIN) is to be found in Maurer, Salomaa and Wood [1980b]. 
Ginsburg, Leong, Mayer and Wotschke [1979] provides the bas is for Section II.5.1. Precedence relations are the subject of Ginsburg and Wood [1978] and the basic material on pushdown acceptor forms is to be found in Ginsburg and Spanier [1978] (see also Wood [1978]). The work on parsing has been followed up for EOL forms in Maurer, Saloma and Wood [1980c] and that for pushdown forms in Ginsburg and Kintala [1979].

Finally Section 11.6 covers recent work on collections of language families defined by generative devices. The material for this section is taken from Maurer, Salomaa and Wood [1979c, 1980e,f]. other papers in this area are Maurer, Salomaa and Wood [1980h,i] and ottmann, Saloma and Wood [1979]. In particular Maurer, Salomaa and Wood [1980h] derives an important relationship between s-interpretations of finite forms and graphs. This is used to solve an important problem concerning finite forms. In Maurer, Salomaa and Wood [1980j] it is shown that there exists a dense pair of finite forms. This is based on the result of Welzl [1980], who has proved a similar result for graph families.

Chapter III: The notion of an EOL form was first discussed in May 1975 by Maurer, Salomaa and myself and this led to the first paper on the topic of EOL forms, namely Maurer, Salomaa and Wood [1977]. The first two sections of this chapter, dealing with EOL and ETOL forms, rely heavily on Maurer, Salomaa and Wood [1977 and 1978b]. Recently Ehrenfeucht, Rozenberg and Verraedt [1980a,b] have shown the decidability of expansion and contraction simulation.

In Section III. 3 the topic of completeness initiated in Maurer, Salomaa and Wood [1977] is presented. The completeness of two-symbol EPOL forms is a simplified exposition of Culik II, Maurer and 0ttmann [1978], while the completeness of $\{S, A, a\}$-synchro-EPOL forms follows that of Maurer, Salomaa and Wood [1980c]. Under g-interpretations the completeness of grammar forms, in particular two-symbol forms is essentially straightforward, however under s-interpretations even the two-symbol case has not been fully resolved, see Maurer, Salomaa and Wood [1978f and 1980b]. The resolution of context-free completeness for two-symbol grammar forms depends upon a positive solution to the Great Lemma Conjecture (Maurer, Saloma and Wood [1978f] and Wood [1979]), which is a nontrivial, but reasonable, extension of the supernromal form theorem, see section II.4.1. 
Section III.3.3 discusses ETOL forms for which little is known (see Maurer, Salomaa and Wood [1978b] and Skyum [1978]) and Section III.3.4 considers regular-completeness of EOL forms and is taken from Maurer, Salomaa and Wood [1977]. The study of very completeness (vompleteness) and goodness in section III.4, which is a natural follow-up to the study of completeness is based on the investigations reported in Maurer, Salomaa and Wood [1978c,d]. The material on good ETOL forms is based on Skyum [1978]. In Hule, Maurer and Ottmann [1978] this concept is studied for OL forms.

Decidability issues are discussed in Section III.5. The decidability result for strong form equivalence was first proved in Cremers and Ginsburg [1975], the presentation here is new. The NP-completeness result follows that in Hunt III and Rosenkrantz [1977], this result has been strengthened considerably by Maurer and Sudborough [1980] who have shown that whether or not an arbitrarily given graph is an interpretation of the complete graph with three nodes is NP-complete. Maurer, Salomaa and Wood [1978a] is the source for the results on uniform interpretations and undecidability and the results on $O L$ forms are based on those in Maurer, ottmann and Salomaa [1977].

other decidability results can be found in Albert, Maurer and Ottmann [1978], Albert, Maurer and Rozenberg [1979], Blattner [1979b], Culik II, Maurer, Ottmann, Ruohonen and Salomaa [1978], Ginsburg and Spanier [1979a,b], Maurer and Rozenberg [1980], Maurer, Salomaa and Wood [1979c and 1980b] and Ottmann, Salomaa and Wood [1979]. These are discussed in more detail in the following section. Finally Section III.6.7 is based on Maurer, Salomaa and Wood [1977], Section III.6.2 on Maurer, Salomaa and Wood [1980c], Section III.6.3 on ATbert, Maurer and Rozenberg [1979] and Section III.6.4 on Maurer, Salomaa and Wood [1980a]. A very important result, which has been omitted is that of Albert and Maurer [1978], which demonstrates that no EOL form family equals $\mathcal{L}(\mathrm{CF})$. Other results of interest are to be found in Albert, Maurer and 0ttmann [1978], where regular-bounded $O L$ forms are studied and Maurer and Rozenberg [1980], where context-free-bounded EOL forms are studied. 
CHAPTER IV: Cremers and Ginsburg [1975] not only introduced contextfree grammar forms but also phrase structure grammar forms. In Ginsburg [1978] the question, "Is there a g-grammatical family properly in between $\mathcal{L}(C F)$ and $\mathcal{L}(R E)$ ?" was raised and moreover is still open (and remains so at the time of writing). Maurer, Penttonen, Salomaa and Wood [1979] contains the basic results of Section IV.1. The notion of "smoothness" of a phrase structure form is taken from Maurer, Salomaa and Wood [1979b] where the generation of various well known families is discussed. An interesting and early result on context dependent grammar forms is to be found in Maurer and Wood [1976].

Raija Leipula first introduced and investigated the matrix forms studied in Section IV. 3 in her licentiate thesis and a Tso in her doctoral thesis, see Leipala [1977, 1978, 1979a and 1979b]. Dassow [1979] has recentiy been investigating programmed grammar forms.

Finally, the discussion in section II.4 on controlled grammar forms is based on the work of Sheila Greibach [1977], who initiated and carried out a major study of controlled grammar forms, which she followed up in Greibach [1978]. A recent paper on Szilard languages of grammar forms is Maurer, Saloma and Wood [1980g]. 
V.2 A Discussion of Some Omitted Results

Every author selects the material he wishes to present and omits that which he doesn't. The present author is no exception to this rule. Some material has been omitted for lack of space, some because it is too new, some because it isn't of interest to me and some because I feel unable to do it justice.

In the following some of the omitted material will be discussed briefly. I should stress that the ordering of results is fortuitous rather than a ranking in order of importance. However two results stand out. Result (2) is perhaps the most surprising result in that it mirrors the corresponding result for grammars, while result (6) breaks new ground using the correspondence between finite forms and graph forms.

\section{Decidability of Structural Equivalence}

Let $G_{1}$ and $G_{2}$ be two grammar forms under g-interpretation, we say $G_{1}$ and $G_{2}$ are structurally equivalent if for every $G_{1}^{\prime} \underset{g}{G} G_{1}$ there is a $G_{2}^{\prime} \rightarrow G_{2}$ such that $G_{1}^{\prime}$ and $G_{2}^{\prime}$ are structurally equivalent and vice versa. Walter [1977] has shown that structural equivalence of two grammar forms is decidable. This result was the first nontrivial decidability result for grammar forms.

\section{Decidability of Form Equivalence for PDOL Forms}

Let $G=(\Sigma, h, \sigma)$ be a DOL form, that is $h$ is a homomorphism from $\Sigma^{*} \rightarrow \Sigma^{*}$ and $\sigma$ is in $\Sigma^{*}$. We say $G$ is propagating if $h(x) \neq \lambda$ for al1 $x$ in $\Sigma^{+}$. We say a $D 0 L$ form $G^{\prime}=\left(\Sigma^{\prime}, h^{\prime}, \sigma^{\prime}\right)$ is a (deterministic) interpretation of $G$ modulo $\mu$, if $\mu$ is a dfl-substitution, $\mu(a) \subseteq \Sigma^{\prime}$, for all a in $\Sigma, \sigma^{\prime}$ is in $\mu(\sigma)$ and $h\left(a^{\prime}\right)$ is in $\mu(h(a))$ for all $a^{\prime}$ in $\Sigma^{\prime}$ where $a^{\prime}$ is in $\mu(a)$. Surprisingly Culik II, Maurer, Ottmann, Ruohonen and Saloma [1978] have shown:

(a) that two PDOL forms $G_{1}$ and $G_{2}$ are form equivalent iff they are sequence equivalent, where $i t$ is assumed that at least one of $G_{1}$ and $G_{2}$ generates a word of length greater than one. The sequence of a DOL form $G=(\Sigma, h, \sigma)$ is the sequence $E(G)=h^{0}(\sigma)=\sigma$, $h^{1}(\sigma)=h(\sigma), h^{2}(\sigma), \ldots$ and similarly for any interpretation of $G$.

(b) that it is decidable if two PDOL forms $G_{1}$ and $G_{2}$ are form equivalent. This follows from (a) and the fact that if $G_{1}$ and $G_{2}$ are sequence equivalent then $E\left(G_{1}\right)$ and $E\left(G_{2}\right)$ are isomorphic (excluding the special case mentioned above). 


\section{Decidability of Form Equivalence for Grammar Forms}

This result was first claimed in Ginsburg [1978] for ginterpretations which has given rise to Ginsburg and Spanier [1979a,b]. A special case is treated in Ginsburg and Goldstine [1977]. However at the time of writing none of these three manuscripts has been circulated. However Blattner [1979b] has presented a different proof of this decidability result as an extended abstract. This author believes that g-form equivalence is decidable, but he has yet to see a complete and correct proof. A full version of Blattner [1979b] is currently in preparation. For s-interpretations there are only two partial results, namely (a) it is decidable whether or not two sub-regular grammar forms are s-form equivalent, see ottmann, Salomaa and Wood [1979], and (b) it is decidable whether or not two sub-linear twosymbol grammar forms are s-form equivalent, see Maurer, Saloma and Wood $[1979 \mathrm{c}]$.

\section{Ambiguity and Grammatical Families}

Blattner [1979a] considers an interesting way of expressing the notion of inherent ambiguity. Normally we say a language $L$ is (inherently) ambiguous with respect to one of the language families in the chomsky hierarchy if for any grammar $G$ within the corresponding class, which generates $L, G$ is ambiguous. Thus inherent ambiguity is better expressed as $\boldsymbol{\xi}$-ambiguity where $\mathcal{G}$ is a collection of grammars, that is it is ambiguous relative to the class $\mathcal{G}$. Blattner [1979a] uses the g-grammar families to express relative ambiguity and demonstrates that there are $\xi_{g}(G)$-ambiguous languages in every class $\mathcal{L}_{\mathrm{g}}(G, \Rightarrow)$, when $\mathcal{L}_{\mathrm{g}}(G, \Rightarrow)$ properly contains $\mathcal{L}(R E G)$.

\section{Completeness of Grammar Forms and Decidability}

Given a grammar form $G=(V, \Sigma, P, S)$ then it is trivially decidable whether or not $\mathcal{L}_{S}(G, \Rightarrow)$ is contained in $\mathcal{L}$ (FIN). We have shown that whether or not $\mathcal{L}_{S}(G, \Rightarrow)=\mathscr{L}$ (REG) is also decidable using the characterization theorem: $\mathcal{L}_{S}(G, \Rightarrow)=\mathcal{L}(R E G)$ iff $G$ is non-selfembedding and there exists a in $\Sigma$ such that $a^{+} \subseteq L(G, \Rightarrow)$. Recentiy, Maurer, Salomaa and Wood [1980b] have shown that it is decidable whether or not $\mathcal{L}_{S}(G, \Rightarrow)=\mathscr{L}($ LIN $)$. However, the question: "Is $\mathcal{L}_{\mathrm{S}}(G, \Rightarrow)=\mathscr{L}(C F)$ decidable?", is still open, see Maurer, Salomaa and Wood [1978f]. 


\section{Finite Forms and Graph Forms}

Recently Maurer, Salomaa and Wood [1980h] have taken the notion of a graph form, first mentioned in Ginsburg [1978], and by way of the close relationship of graph forms with particular finite grammar forms have derived an important result for finite grammar forms. Now each finite s-grammatical family is characterized by the language of its form. So consider a language $L \subseteq \Sigma^{*}$ where $L$ consists only of two letter words. Then the corresponding digraph $G_{L}$ has $\# \Sigma$ nodes and has an edge $(a, b)$ if $a b$ is in $L$. The obvious definition of s-interpretation for graphs and digraphs implies that a graph $G \underset{S}{\Delta} G_{L}$ iff there exists an $L^{\prime} \rightarrow L$ such that $G_{L^{\prime}}=G$. This interrelationship has led to a proof of the following result:

There exists an infinite sequence of finite grammar forms

$$
G_{1}, G_{2}, \ldots
$$

such that $\mathcal{L}_{S}\left(G_{i}, \Rightarrow\right) \underset{\mathcal{L}_{S}}{\not}\left(G_{i+1}, \Rightarrow\right)$, and there is no

grammar form $G$ such that $\mathcal{Z}_{S}\left(G_{i} \Rightarrow\right) \not \mathcal{Z}_{S}(G, \Rightarrow) \not \mathcal{Z}_{S}\left(G_{j+1}, \Rightarrow\right)$

for al1 $i \geq 1$. The existence of such a descending chain was an open problem in Maurer, Salomaa and Wood [1980e].

Similarly a relationship between graphs and commutative languages can be established, giving rise to graph forms and the so called color families, see Maurer, Salomaa and Wood [1980h]. These are also discussed in Salomaa [1980b]. Welzl [1980] has shown dense intervals exist even in this situation, while Maurer, Salomaa and Wood [1980j] have shown that $(\mathscr{L}(\mathrm{REG}), \mathcal{Z}(\mathrm{CF}))$ is not maximally dense using an approach based on that of welzl.

\section{Descriptional Complexity}

The results given in Section II.3.4 on (production) minimal grammar forms with respect to strong form equivalence are one aspect of descriptional complexity. On the other hand 1ittle work has been done to characterize production minimal grammar forms under form equivalence. In Ginsburg and Lynch [1976] some prelisinary results are given for g-interpretations. They show that left and right linear grammar forms are essentially equal in efficiency. Further each form which generates the regular sets provides at most polynomial improvement over right linear normal form. 
Similar results also hold for forms giving exactly the linear languages, while for those generating exactly the context-free languages only linear improvement is possible.

of course not only production minimality need be studied but also other size measures, for example the total number of symbols, the number of nonterminal occurrences and the total length of all productions. Ginsburg and Lynch [1976] also consider these cases and derive similar results to those for production minimality.

Another approach to descriptional complexity of grammar forms is through derivational complexity, see Ginsburg and Lynch [1977]. Letting $G$ be a grammar form and $G^{\prime}=(V, \Sigma, P, S) \underset{g}{\Delta}$, then the complexity function $d_{G}: \Sigma^{*} \rightarrow \mathbb{N}$ is defined by: $d_{G}(x)=$ the length of the minimal derivation for $x$ in $L\left(G^{\prime}, \Rightarrow\right)$ and $d_{G}(x)$ is undefined if $x$ is not in $L\left(G^{\prime}, \Rightarrow\right)$. Ginsburg and Lynch [1977] show that for any integer $n>0$ there exists $G^{\prime \prime} \vec{g} G$ such that $L\left(G^{\prime \prime}, \Rightarrow\right)=L\left(G^{\prime}, \Rightarrow\right)$ and for all sufficiently large words $x, d_{G^{\prime \prime}}(x) \leq|x| / n$.

In Walter [1975d] some general results on complexity measures for grammar forms are given. But apart from this almost nothing has been done.

\section{Pure Grammars and Forms}

In Maurer, Saloma and Wood [1978e] the following problem was raised:

Let $F$ be defined by the productions $S \rightarrow a ; a \rightarrow a ; a \rightarrow$ aS.

Then is $\mathcal{L}_{u}(F, \Rightarrow \subseteq \mathcal{L}(R E G)$ ?

It is clear that $\mathcal{L}_{S}(F, \Rightarrow) \mathcal{L}(C F)$ since $F^{\prime} \underset{s}{A}$ defined by: $S \rightarrow a ; S \rightarrow b ; a \rightarrow a S ; a \rightarrow b ; b \rightarrow b N ; N \rightarrow b$ generates

$\left\{b^{F(i)}\right.$ : $F(i)$ is the ith Fibonacci number .

Consider any arbitrary uniform interpretation $F^{\prime}=\left(V^{\prime}, \Sigma^{\prime}, P^{\prime}, S^{\prime}\right)$ of $F$, then it follows that for each a in $\Sigma^{\prime}$ there can only be productions of the type $a \rightarrow a$ and $a \rightarrow a A$ in $P^{\prime}$. Now if $a \rightarrow a A$ is the only type of production for a, then any word containing a will not produce any further terminal words according to $F^{\prime}$. Therefore a can only be introduced into a terminal word of $F^{\prime}$ by means of a production of type $A \rightarrow a$. On the other hand if $a \rightarrow$ a is a production for a then whenever a is deposited in a word by a derivation of $F^{\prime}$ it remains in the word from thereon $i n$. 
These preliminary observations lead to the consideration of only those $F^{\prime} A F$ such that for each a in $\Sigma^{\prime}$ there is a production $a \rightarrow a$ in $\mathrm{P}^{\prime}$. This has the effect of "sequentializing" the rewriting according to $F^{\prime}$. We say $F^{\prime}$ is a stable interpretation of $F$ in this case. It can be proved that $\boldsymbol{\alpha}_{u}\left(\overline{F, \Rightarrow} \subseteq \mathcal{Z}(R E G)\right.$ iff $\mathcal{\chi}_{\text {stab }}(F, \Rightarrow \subseteq \mathcal{Z}(R E G)$. And if we now treat $F$ as an $O L$ form $\bar{F}$ it can also be shown that $\mathcal{L}_{\text {stab }}(F, \Rightarrow) \subseteq \mathcal{L}(R E G)$ iff $\mathcal{L}_{\text {stab }}(\bar{F}, \Rightarrow) \subseteq \mathcal{L}(R E G)$. But when $F$ and its interpretations are treated as $0 \mathrm{~L}$ forms, the stable interpretation means we can further reduce our question to:

Let $G$ be defined by $a \rightarrow a b, b \rightarrow a$ where $b$ is the sentence symbol, where $G$ is now a context-free pure grammar (no nonterminals), then is $\mathcal{L}_{u}(G, \Rightarrow) \subseteq \mathcal{L}(R E G)$ ?

Hagauer [1978] showed that this is not the case and hence $\mathcal{L}_{u}(F, \Rightarrow \notin \mathcal{L}(R E G)$ also. This reduction served however to demonstrate that 1 ittle was known about pure grammars apart from the early work of Buchi [1964], Gabrielian [1980], originally issued as a technical report in 1970, and Buttlemann, Pyster and Reeker [1974]. Since this time Maurer, Salomaa and Wood [1980d] and Ehrenfeucht and Rozenberg [1979; 1980a,b,c,d] have begun to correct this inbalance. Much remains to be done however. Some preliminary results on pure grammar forms are to be found in Vaishnavi and Wood [1978]. Hagauer is currently completing a thesis on the topic of pure grammars and pure grammar forms. Recentiy he has shown the decidability of form equivalence for deterministic pure grammar forms, Hagauer [1980a].

\section{Syntax Specification}

An interesting application of grammar forms can be found in Ginsburg and Rounds [1978]. They consider dynamic syntax specification, that is a context-free grammar is given in advance which is modified during the parsing process to produce a specific grammar for the specific input. This ensures that much of the typical contextsensitive portions of programming language specification, for example valid identifier usage, can be dynamically included in the derived grammar.

The main contribution of Ginsburg and Rounds [1978] is to notice that the derived grammar is an interpretation of the original grammar. Hence the derivation can be construed as constructing an interpretation dynamically. 


\section{Subset-strict Interpretations}

Miles [1978] considers a variant of s-interpretations for which a specified subset of the terminals of the grammar form are s-interpreted while the remainder are g-interpreted. This provides a a unified framework for a study of the effect of punctuation or markers upon ambiguity, for example the unambiguity of the parenthesis languages.

\section{Grammatical Family Characterizations}

Under g-interpretations each nontrivial g-grammatical family other than the finite sets is a full principal semi-AFL. One natural question raised by this fact is: When is a context-free full principal semi-AFL also a g-grammatical family?

Some preliminary and interesting characterization results are available in Cremers, Ginsburg and Spanier [1977], but these are not completely satisfactory.

\section{The Empty Word}

In Maurer, Saloma and Wood [1977] and in subsequent papers the so-called $\lambda$-convention is invoked. This convention is also assumed in the present book. Simply stated it says:

Two languages are considered equal if they differ by at most the empty word. Two language families are considered equal if for each language different from the empty set and $\{\lambda\}$ in one family a language differing by at most the empty word can be found in the other family and vice versa.

Thus most of the time the empty word can be ignored, however, there are situations when it cannot be. Thus in section III.2. we have shown that for the EOL form $F$ :

$$
S \rightarrow a ; a \rightarrow a b b a ; b \rightarrow \lambda
$$

there is no form equivalent $\lambda$-free EOL form. In particular this implies there is no $\lambda$-free vomplete EOL form (see section III.4.2). Recently Ainhirn and Maurer [1979] have improved this result to:

There is no vomplete EOL form which has only $\lambda$-productions for terminals. In other words $\lambda$-productions for nonterminals are a necessity for vomplete EOL forms.

In this book we have avoided a number of troublesome situations for EOL forms by only dealing with the subclass of propagating $E O L$ forms. 
Another example of the difficulties of the empty word is to be found in Maurer, Saloma and Wood [1977, p. 87] in the invalid proof of Theorem 5.1. As in Section III.3.1 the notion of a corresponding EDTOL grammar of an EOL grammar is given. Moreover it is shown that if a propagating EOL grammar $F$ generates $\left\{a^{i} b^{i} c^{i}: i \geq 1\right\}$ then the corresponding propagating EDTOL grammar $G$ also generates this language. Moreover the EDTOL grammar $G$ must be looping and hence $F$ is also looping. The propagating assumption is necessary, since if $F$ and hence $G$ are not propagating, then $G$ need not be looping.

Further study of the empty word seems indicated by these two examples.

\section{Psuedo-termina7s}

In Culik II, Maurer and Ottmann [1978] completeness of twosymbol EOL forms is studied in some detail. However only a partial characterization of complete two-symbol EOL forms is given (at the time of writing it remains partial). A number of results contained in this paper (see also section III.3.1) are dependent on the fact that in EOL grammars terminals may be used without ever appearing in any terminal word. Such terminals are designated as pseudo-terminals. When studying the proofs in Culik II, Maurer and 0ttmann [1978] one gets the feeling that pseudo-terminals are cheating in some way.

one can define terminals in a much more restrictive manner, namely a symbol in an EOL grammar is designated as a real terminal only if it appears in some word of the language of the grammar. Clearly EOL grammars, which only have real terminals can be defined and also interpretations can be restricted to such grammars.

Ainhirn [1979] has shown recently that when attention is restricted to two-symbol EOL forms, which only allow interpretation grammars to have real terminals, then completeness can be fully characterized.

\section{Morphisms}

As mentioned in section 1.3 grammar morphisms provide the most general framework for a discussion of grammatical similarity. Recent work of walter and his coworkers has demonstrated a number of generalizations of results in form theory. It remains to be seen whether grammar morphisms are in fact the most appropriate tool for investigating grammatical similarity. 
The interested reader should consult 0chsenschlager and Pfliiger [1978], Walter [1976 and 1979], Walter, Kekiikoglou and Kern [1979], and Ross, Hotz and Benson [1978]. Some preliminary results for L morphisms were given by Lange [1978] while independently Kobuchit and his co-workers have considered a special case of $L$ morphism called simulation. See Kobuchi [1977], Kobuchi and Culik II [1980], Kobuchi and Seki [1979], Kobuchi and Wood [1980] and Seki and Kobuchi [1980]. 


\section{V.3 New Directions and Open Problems}

This section is intentionally short since I do not find the role of prophet an easy one. It is very easy to list many open problems. However it must be borne in mind that it is not an open problem per se which is important, but rather the context in which it is asked, since this gives it meaning in some overall investigation. For example the super-normal form result (Theorem II.4.10) is not only yet another normal form theorem for context-free grammars but it adds to the understanding of other normal forms. For example it answers one simple question about Greibach two-standard form, namely, if productions of the type:

(1) $A \rightarrow B C$ and $A \rightarrow a$

are sufficient to generate all of $\mathcal{K}(C F)$, then why are productions of the type:

(2) $A \rightarrow a B C$ and $A \rightarrow a$

not sufficient to do this. The answer is simply that even length words cannot be generated by a context-free grammar of type (2), however if we allow

(3) $A \rightarrow a B C ; A \rightarrow a B$ and $A \rightarrow a$

as is usually done we do obtain $\mathcal{L}(C F)$. On the other hand the supernormal form theorem tells us that

(4) $A \rightarrow a B C ; A \rightarrow a ; A \rightarrow a b$

types of productions are also sufficient. Thus productions of type $A \rightarrow a B$

are not really necessary at all to obtain a Greibach-like two-standard normal form theorem for context-free grammars.

This example in a small way illustrates my point; out of its context the super-normal form result is next to meaningless. However in its context it has much to tell us about context-free grammar forms which are similar with respect to their being able to generate all context-free languages and to explain why certain types of productions are or are not able to give normal forms for context-free grammars.

As mentioned in Chapter I. I consider that the study of grammar and $L$ forms provides a threefold contribution to formal 1anguage theory:

(a) giving a better understanding of generative devices,

(b) giving a deeper understanding of the families of context-free and EOL languages, and

(c) giving another approach to a theory of grammatical similarity. 
It is, of course, possible that form theory is a red herring as far as these aims are concerned, however the results obtained to date lead me to believe that this is not the case. Three relatively new directions are evident at present.

First, matrix forms as studied by Raija Leipald [1977, 1978 and $1979 \mathrm{a}, \mathrm{b}]$. In her continuing study of the generation of the context-free languages by such forms she has shed new light on matrix grammars and their Ianguages.

clearly programmed forms, indexed forms and other such forms variants of typical extensions of context-free grammars can be studied. Whether this is a useful exercise remains to be seen. Dassow [1979] has some preliminary results on programmed forms.

Second, controlled grammar forms as studied by sheila Greibach [1977] and 1978]. When comparing sequential and parallel rewriting the first obvious divergence (namely sequential rewriting implies that a chaice of which symbol to rewrite has to be made, while for parallel rewriting no such choice need be made since all symbols must be rewritten) has far reaching consequences. Greibach's results on left-derivation-bounded controlled grammar forms tends to confirm this conclusion. The fact that she has also studied grammar families primarily as operators on language families rather than as controlled grammatical families in their own right has also meant her work gives a deeper understanding of the context-free and recursively enumerable languages.

Third, the notion of dense hierarchies as studied by Maurer, Salomaa and Wood [1979c and $1980 e, h, i, j]$ and 0 ttmann, Salomaa and Wood [1979] (see Section II.6). These preliminary results lead to some further understanding of the structure of the family of contextfree languages. This is especially true since surprisingly little is known about the collections of context-free semi-AFL's or AFL's, for example. A tantalizing open problem of whether or not there exists a dense pair of finite forms was recently solved in Maurer, Salomaa and Wood [1980j] by way of a technique due to Welz] [1980].

Apart from these three "new" directions there is still much to discover in the area of g-interpretations of grammar forms and $s-$ interpretations of grammar forms and EOL forms.

Before listing a number of open problems, we mention two areas in formal 1 ariguage theory which have gained prominence because of work in form theory. 
First, in Maurer, Salomaa and Wood [1979b] a question about a specific synchro-EOL form under uniform interpretations was reduced to whether a corresponding pure context-free grammar (a context-free grammar with only terminals) generated a regular language. Surprisingly little was known, since there were so few results in this area. Vet the study of pure grammars must surely be basic to any study of sequential rewriting. This observation led initially to Maurer, Salomaa and Wood [1980d] and Hagauer [1978], which was followed by Ehrenfeucht and Rozenberg [1979; 1980a,b,c) and by Hagauer [1980a].

Second, in Maurer, Saloma and Wood [1980c] a preliminary study of parsing led to questions about ambiguity for EOL grammars. Some basic results are given in this paper, while a number of open questions have been recently answered in Ehrenfeucht and Rozenberg $[1980 \mathrm{e}, \mathrm{f}]$.

\section{Problems}

The following problems are not 1 isted in any order of difficulty. An '*' indicates that the problem or one closely related to it was mentioned in section 2 .

*1) Given two DOL forms (or DTOL forms) $F_{1}$ and $F_{2}$ is it decidable whether or not $\mathcal{L}_{5}\left(F_{1}, \Rightarrow\right)=\mathcal{L}_{s}\left(F_{2}, \Rightarrow\right.$ '?

2) Given two EOL forms $F_{1}$ and $F_{2}$ is it decidable whether or not $\mathcal{L}_{s}\left(F_{1}, \Rightarrow\right)=\mathscr{L}_{s}\left(F_{2}, \Rightarrow\right)$ ? We conjecture: no.

*3) Given two grammar forms $F_{1}$ and $F_{2}$ it is decidable whether or not $\mathcal{L}_{s}\left(F_{1} \Rightarrow\right)=\mathcal{L}_{s}\left(F_{2}, \Rightarrow\right)$ ? We conjecture: no.

Note that if (i) both $F_{1}$ and $F_{2}$ are sub-regular it is decidable by Ottmann, Salomaa and Wood [1979] and (ii) both $F_{1}$ and $F_{2}$ are sublinear two-symbol forms then it is decidable by Maurer, Saloma and Wood [1979c]. The two-symbol case can be resolved completely if the following conjecture can be proved.

4) Let $M$ be an ultimately periodic set of positive integers, then by $\mathcal{L}$ (CF,M) we denote the family of all context-free languages whose length set is contained in $M$. 
Conjecture: The Great Lemma

Let $G$ be a two-symbol expansive grammar form. Then $\mathcal{L}_{S}(G, \Rightarrow)=\mathcal{L}(C F, L S(G, \Rightarrow))$.

This is known to be true if $G$ is cofinite. It is also true if $L S(G, \Rightarrow)$ equals all positive integers. We conjecture that

it is true for all two-symbol expansive $G$.

5) Let $F=(V,\{a\}, P, S)$ be a synchronized EOL form. Is it decidable whether or not $L(F, \Rightarrow)=a^{*}$ ? Conjecture: yes.

This will imply it is decidable whether or not a synchro-EOL form is regular-sufficient.

6) Is it decidable whether or not an EOL form is complete or vomplete.

7) Is it decidable whether or not two EOL forms are mutually good?

8) For an EOL form $F$ and a positive integer $i$ define:

$\mathcal{L}_{s}(i)\left(F, \Rightarrow\right.$ to be either $\mathcal{L}_{s}(F, \Rightarrow$ if $i=1$ or

$\left\{\mathcal{L}_{S}(i-1)\left(F^{\prime}, \Rightarrow\right): F^{\prime} \underset{s}{\Delta} F\right\}$ otherwise.

Do there exist EOL forms $F_{1}$ and $F_{2}$ such that

$L\left(F_{1}, \Rightarrow\right)=L\left(F_{2}, \Rightarrow\right), \mathcal{L}_{s}\left(F_{1}, \Rightarrow\right)=\mathcal{L}_{s}^{2}\left(F_{2}, \Rightarrow\right)$,

$\mathcal{L}_{s}^{(2)}\left(F_{1}, \Rightarrow\right)=\mathcal{L}_{s}^{(2)}\left(F_{2}, \Rightarrow\right.$ but $\mathcal{L}_{s}^{(3)}\left(F_{1}, \Rightarrow \neq \mathcal{L}_{s}(3)\left(F_{2}, \Rightarrow\right.\right.$ ?

This question is related to the notion of mutual goodness, see Section III.4.4. Is it possible to exhibit an $F_{1}$ and $F_{2}$ such that differentiation only takes place at level $n$, for arbitrary $n>0$ ?

9) Characterize the family of good languages, see section III.4.4.

10) Given an ETOL form $F$ is it decidable whether or not $F$ is regularsufficient or context-free-sufficient. Similarly for regularand context-free-boundedness.

11) We say a grammar form $G$ has a successor if there exists a grammar form $H$ such that $\mathcal{L}_{s}(G, \Rightarrow) \subsetneq \mathcal{L}_{s}(H, \Rightarrow)$ and there is no other $s$-grammatical family in between. Is it decidable whether or not a finite grammar form has a successor?

12) Given two pure context-free grammars it is decidable whether or not they generate the same collection of sequences, see Ehrenfeucht and Rozenberg [1980]. Does a similar result hold for pure context-free grammar forms?

13) Consider a grammar form $F$ and a language which you are told is in $\mathcal{L}_{S}(F, \Rightarrow)$. It is possible to construct an $F^{\prime} \Delta_{S} F$ such that $L\left(F^{\prime}, \Rightarrow\right)=L$ ? This is one example of an inference problem. 
14) Is every sub-regular synchro-EOL form family a grammatical family?

15) It is easy to show that $\mathcal{L}(\mathrm{OL}) \subseteq \mathcal{L}_{\mathrm{s}}(\mathrm{F}, \Rightarrow$ implies $\mathcal{L}(E O L)=\mathcal{L}_{s}(F, \Rightarrow)$, if $F$ is a synchro-EOL form. Is this true in general?

16) Let $F$ be a synchro-EOL form. Find necessary and sufficient conditions for $\mathcal{L}(C F) \subseteq \mathcal{L}_{S}(F, \Rightarrow)$.

17) Let $G$ be a grammar form. Find necessary and sufficient conditions on $G$ such that $\mathcal{L}_{\mathrm{g}}(G, \Rightarrow)$ equals the full semi-AFL generated by $L(G, \Rightarrow)$.

18) Characterize those context-free full principal semi-AFL's which are g-grammatical famijies.

19) For two grammar forms $G_{1}$ and $G_{2}$ is $\mathcal{L}_{g}\left(G_{1}, \Rightarrow\right) \cap \mathcal{L}_{g}\left(G_{2}, \Rightarrow\right)$ $=\{L: L$ is in both families $\}$ always g-grammatical? If not, when is it and is it decidable?

20) For a given context-free language $L$ is there a "smallest" g-gramatical family containing it?

21) Let $G$ be a grammar form and $\mathcal{L}(C F) \subseteq \mathcal{L}_{g}(G, \Rightarrow) \subseteq \mathcal{L}(R E)$. Then it is conjectured (see section IV.1) that either $\mathcal{L}_{\mathrm{g}}(G, \Rightarrow)=\mathcal{L}$ (CF) or it equals $\mathcal{L}(R E)$. Prove or disprove this conjecture.

22) Given two dpda forms $M_{1}$ and $M_{2}$ is it decidable whether or not $\mathcal{L}_{s}\left(M_{1}, \Rightarrow\right)=\mathcal{L}_{s}\left(M_{2}, \Rightarrow\right)$ ? This has a flavour similar to the PDOL form equivalence problem.

23) It is conjectured that $\mathcal{L}$ (LIN) has no generator. Prove or disprove this (see Section III.6.4).

24) It is conjectured that for an EOL form $F$ whenever $\mathcal{L}($ LIN $) \underset{\neq}{\mp}\left(F, \Rightarrow\right.$ then $\mathcal{L}_{s}(F, \Rightarrow$ contains non-context-free languages. Prove or disprove this. 